\title{
Energy-Efficient Analog Beamforming with Short Packets in Millimeter-Wave MIMO Systems
}

\author{
Jordi Borras*, Francesc Molina*, Roberto López-Valcarce ${ }^{\dagger}$, and Josep Sala-Álvarez* \\ *Technical University of Catalonia, Spain \\ ${ }^{\dagger}$ atlanTTic Research Center, University of Vigo, Spain \\ Email: jordi.borras.pino@upc.edu, francesc.molina@upc.edu, valcarce@gts.uvigo.es, josep.sala@upc.edu
}

\begin{abstract}
The energy-aware design of fully analog precoders and combiners is investigated in this paper. To that aim, we consider a millimeter-wave short-packet communication scenario where power consumption and hardware complexity constitute relevant factors. User terminals employ a single channel encoder and, at hardware level, they implement analog architectures that combine phase shifters and switches. Additionally, transmitting terminals also leverage a power control system. An energy efficiency figure of merit is considered, and its optimization subject to finite packet-length constraints is tackled cyclically over disconnected feasible sets. Numerical results are reported firstly, to illustrate the advantages of the proposed short-packet fully analog design, secondly, to compare its performance to that of on-off beamforming, and finally, to discuss the optimality of proposed optimization algorithm.
\end{abstract}

Index Terms-Analog beamforming, short-packet communication, energy efficiency, packet error rate, mmWave MIMO.

\section{INTRODUCTION}

The exponential growth of simultaneously connected devices envisaged in Internet of Things (IoT) [1] and machinetype communications (MTC) [2] settings, and the high demanding user requirements [3], have boosted the interest on (large) multi-antenna schemes. Yet, the power consumption of such systems in the mmWave band poses severe limitations to MTC devices due to their simple hardware architectures [4].

Hybrid precoding (or beamforming) [5] emerges as a promising solution to overcome the aforementioned limitations. A number of works deal with the design of precoding/combining schemes when the analog part is composed of phase shifters [6-10] and/or switches [11-14]. Typically, these works tackle the sum-rate optimization subject to power constraints. Notwithstanding, in the context of IoT and MTC, some considerations need to be taken into account. Firstly, the joint design problem from the energy efficiency (EE) point of view may be more appropriate for cost-effective low-power terminals. Secondly, the asymptotic-packet limit may be of

J. Borras, F. Molina and J. Sala-Álvarez are supported in part by Agencia Estatal de Investigación (AEI, Spain) and by European Regional Development Fund (ERDF) under projects WINTER (TEC2016-76409-C2-1-R) and RODIN (PID2019-105717RB-C22), and in part by the Catalan Government (AGAUR) through Grant 2017 SGR 578. J. Borras is also supported by Fellowship FPI BES-2017-080071 (Spanish Government and ERDF). F. Molina is also supported by Fellowship 2019FI_B1 00085 (Catalan Government, AGAUR).

R. López-Valcarce is supported in part by AEI (Spain) and by ERDF under projects WINTER (TEC2016-76409-C2-2-R) and RODIN (PID2019105717RB-C21), and in part by Xunta de Galicia (Agrupación Estratéxica Consolidada de Galicia accreditation 2016-2019).

All authors are also supported by the AEI and ERDF through the research network COMONSENS Network (RED2018-102668-T). moderate/low practical interest since its performance is still far from that attainable under short packets [15], specially so, when systems operate at low signal-to-noise ratios (SNRs).

Some works study the impact on the spectral efficiency of disconnecting phase shifters [16], [17], typically constraining the power consumption. Another approach is based on subconnected analog or hybrid beamforming architectures [18], [19]. The latter can be also found in the literature under the concept of energy-efficient sub-arraying (see, e.g. [20]). In contrast to the former works, [21] addresses the beamformer design from an EE point of view. Therein, closed-form expressions for the asymptotic-packet EE are derived. Yet, the most interesting conclusion is that the combination of phase shifters and switches provides higher EE, while keeping SE, than using only phase shifters. The latter motivates the use of hardware architectures that combine both phase shifters and switches.

In this work, we consider a point-to-point narrowband link in the mmWave band where EE is the chosen key magnitude for system performance evaluation. We consider low complexity terminals which use a single channel encoder and employ single stream transmission with fully analog architectures that combine phase shifters, switches and transmitter power amplifiers with variable gain. In contrast to many works, we focus the discussion on the short-packet (finite blocklength) regime, where the adopted channel encoder-decoder is characterized by the known packet error rate (PER) versus SNR function. In the context of massive access scenarios, as IoT or MTC, several authors have discussed the practical interest of short packets given their reduced latency (see, for instance, [22-24]).

The novelty introduced in this paper relies on the use of packet-length constraints to design energy-efficient ana$\log$ beamforming architectures. We consider the transceivers operating under a maximum error rate, and minimize the total power consumption. Specifically, we design the precoder, combiner and power control subject to per-antenna power constraints, hardware insertion losses, and full channel state information. We consider, for direct application, phase shifters with quantized angular resolution and discrete-level power amplifiers, and solve the non-convex proposal under a cyclic approach with affordable computational complexity.

This paper is organized as follows. In Section II we present the system model. The joint design of fully analog architecture is addressed in Section III. A numerical analysis is reported in Section IV, and the paper is concluded in Section V. 


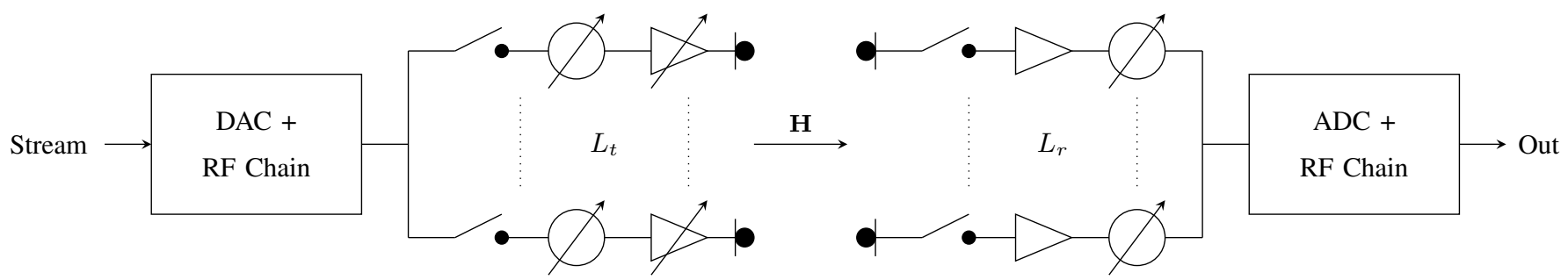

Fig. 1: Point-to-point analog beamforming scenario with phase shifters, switches and variable-gain power amplifiers at transmitter.

\section{SySTEM MODEL}

We envisage a single-stream device-to-device transmission using fully analog beamforming and combining through a mmWave channel. Transmitter and receiver are equipped with $L_{t}$ and $L_{r}$ antennas, respectively. The respective beamformer and combiner vectors can be written as

$$
\mathbf{f} \in \mathbb{C}^{L_{t}} \text { and } \mathbf{w} \in \mathbb{C}^{L_{r}} .
$$

We consider a narrowband flat-fading channel; extensions to wideband frequency-selective scenarios will be the topic of future work. The multipath channel model is given by the channel matrix $\mathbf{H}$, composed by the sum of $N_{\mathrm{cl}}$ clusters of scatterers with $N_{\text {ray }}$ propagation paths in each cluster. The channel matrix is

$$
\mathbf{H}=\sum_{k=1}^{N_{\mathrm{cl}}} \sum_{l=1}^{N_{\mathrm{ray}}} \beta_{k, l} \mathbf{a}_{r}(k, l) \mathbf{a}_{t}^{H}(k, l),
$$

where $\beta_{k, l}$ are the complex channel gains of each channel ray and cluster, and $\mathbf{a}_{t}, \mathbf{a}_{r}$ the steering vectors associated with transmitter and receiver array architectures. Letting $x(t)$ be the transmitted signal, the received signal after down conversion is given by

$$
y(t)=\sqrt{\gamma} \cdot \mathbf{w}^{H} \mathbf{H f} \cdot x(t)+\mathbf{w}^{H} \mathbf{n}(t),
$$

with $\gamma$ denoted pre-combining SNR when $\operatorname{Tr}\left(\mathbf{H}^{H} \mathbf{H}\right)=L_{t} L_{r}$, and $\mathbf{n}(t) \sim \mathcal{N}_{\mathbb{C}}\left(\mathbf{0}, \sigma_{n}^{2} \mathbf{I}_{L_{r}}\right)$ the circularly-symmetric Gaussian noise with variance $\sigma_{n}^{2}$. In the context of hybrid beamforming, and particularly analog beamforming, the joint design of precoder $\mathbf{f}$ and combiner $\mathbf{w}$ under some power constraint and alphabet constraints is typically cast as

$$
\max _{\{\mathbf{f}, \mathbf{w}\}} \log (1+\Gamma) \text {, s.t. } \mathbf{f} \in \mathcal{F}, \mathbf{w} \in \mathcal{W},
$$

where $\mathcal{F}$ and $\mathcal{W}$ stand for the feasible set for precoder and combiner, respectively, and $\Gamma$ denotes the SNR after combining. Notice that, the design problem in (4) under perfect channel state information (CSI) is equivalent to maximizing the SNR after combining defined as

$$
\Gamma \triangleq \frac{\left|\mathbf{w}^{H} \mathbf{H f}\right|^{2}}{\mathbf{w}^{H} \mathbf{w}} \cdot \gamma
$$

where, for simplicity, we assumed $\mathbb{E}\left[|x(t)|^{2}\right] \cdot \sigma_{n}^{-2}=1$.

Nevertheless, the mentioned approach may be only reasonable when the length of transmitted data packets is unboundedly large and the transmitter is equipped with a large bank of capacity-achieving channel codes to select the appropriate encoding rate as a function of the instantaneous channel state. It is worth noting that the latter is too optimistic in the context of IoT and MTC, where user terminals have, in general, simple hardware architectures. Consequently, in our analysis, we consider the transmission of short packets of $n$ symbols at the fixed transmission rate of $R$ bit per channel use employing a single channel encoder. The system SE accounting for the number of effective transmitted bits per channel use is given by the known PER versus SNR characteristic of the employed encoder-decoder pair PER $[\Gamma]$, an univariate non-increasing function of the SNR $\Gamma$. The system spectral efficiency is

$$
\mathrm{SE}[\mathrm{bit} / \mathrm{c} . \mathrm{u}] \triangleq R \cdot(1-\mathrm{PER}[\Gamma]) .
$$

\section{A. Analog Beamforming Architecture}

The considered two-node analog beamforming architecture is that illustrated in Fig. 1. Each antenna of the transmitting terminal is equipped with a switch, a phase shifter and a variable-gain power amplifier (PA). In contrast, the $L_{r}$ antennas of the receiving user terminal have a switch, a phase shifter and a low-noise amplifier (LNA) with fixed power gain $G_{\text {lna }}$. Hence, the precoder and combiner can be expressed as

$$
\begin{aligned}
\mathbf{f} \triangleq L_{\mathrm{sw}} L_{\mathrm{ps}} \cdot \boldsymbol{\beta}_{t} \odot \boldsymbol{\phi}_{t} \odot \boldsymbol{\alpha}, \\
\mathbf{w} \triangleq L_{\mathrm{sw}} L_{\mathrm{ps}} \cdot G_{\mathrm{lna}}^{1 / 2} \cdot \boldsymbol{\beta}_{r} \odot \boldsymbol{\phi}_{r},
\end{aligned}
$$

with $\odot$ the Schur-Hadamard product, $\boldsymbol{\beta}_{t} \in \mathcal{B}^{L_{t}}$ and $\boldsymbol{\beta}_{r} \in \mathcal{B}^{L_{r}}$ Boolean vectors indicating when switches are active,

$$
\begin{aligned}
\boldsymbol{\alpha} & =\left[\alpha_{1}, \alpha_{2}, \ldots, \alpha_{L_{t}}\right]^{T}, \\
\phi_{t} & =\left[\phi_{1}^{t}, \phi_{2}^{t}, \ldots, \phi_{L_{t}}^{t}\right]^{T}, \\
\phi_{r} & =\left[\phi_{1}^{r}, \phi_{2}^{r}, \ldots, \phi_{L_{r}}^{r}\right]^{T},
\end{aligned}
$$

with $\boldsymbol{\alpha}$ the amplitude gain of each antenna, and $\phi_{t}$ and $\phi_{r}$ are the phase-shifting vectors, with unit-magnitude entries. As a last remark on (7)-(8), $L_{\mathrm{sw}}$ and $L_{\mathrm{ps}}$ are the amplitude insertion losses associated with switches and phase shifters, respectively.

For the sake of concreteness, in this work we consider quantized values for both the amplitude gain and phase shifters belonging to the following discrete alphabets:

$$
\begin{aligned}
\mathcal{A} & =\left\{a_{1}, a_{2}, \ldots, a_{K}\right\}, \\
\mathcal{S}_{t} & =\left\{\exp \left(j s_{1}^{t}\right), \exp \left(j s_{2}^{t}\right), \ldots, \exp \left(j s_{2^{b_{t}}}^{t}\right)\right\}, \\
\mathcal{S}_{r} & =\left\{\exp \left(j s_{1}^{r}\right), \exp \left(j s_{2}^{r}\right), \ldots, \exp \left(j s_{2^{b_{r}}}^{r}\right)\right\},
\end{aligned}
$$

where $b_{t}$ and $b_{r}$ are the number of quantization bits considered at each user terminal for the phase shifters, and $K$ the size of the pool of feasible PA gains. In (12), $\mathcal{A}$ is an ordered set with $a_{K} \triangleq \sqrt{P_{0}}$ and $P_{0}$ the per-antenna power constraint. 


\section{ANALOg BeAmFormer DESIGN}

The system SE and power consumption are the relevant key performance indicators in our design. The former is of interest to establish communication between simple machineto-machine terminals while avoiding ineffective energy expenditure. For this reason, we opt for the following EE figure of merit that accounts for the number of bits per Joule during packet transmission in bandwidth $B$ [20]

$$
\mathrm{EE}[\mathrm{bit} / \mathrm{Joule}]=\frac{B \cdot \mathrm{SE}}{P_{\text {tot }}}=B \cdot R \cdot \frac{1-\mathrm{PER}[\Gamma]}{P_{\mathrm{tot}}} .
$$

$P_{\text {tot }}$ stands for the total power consumption. We describe in Section III-A the model adopted for $P_{\text {tot }}$, and the proposed and performed optimizations in Sections III-B and III-C.

\section{A. Power Consumption Model}

We are interested, as shown in Section IV, in comparing the performance of the fully and on-off analog architectures. The adopted power consumption model is relevant for such comparison. We consider an end-to-end power model that accounts for the individual consumed and dissipated powers at both sides. Since the transmitter is endowed with power adaptation, and transmit PAs tend to consume significantly more power than receive LNAs, we neglect the contribution of the latter to the end-to-end power consumption. Henceforth, we assume unit gain LNA, i.e. $G_{\text {lna }}=1$.

Consequently, we adopt the following model where $P_{\text {tot }}$ is decomposed as the sum of the power during packet transmission $P_{\text {data }}$ plus the power consumed by the front-end analog architectures $P_{\mathrm{c}}$,

$$
P_{\text {tot }} \triangleq P_{\text {data }}+P_{\text {c }}
$$

$P_{\text {data }}$ is given by the number of active switches and by the suplied power of each PA, whereas $P_{\mathrm{c}}$ depends on the number of active switches at both front-ends $\left(\|\mathbf{f}\|_{0},\|\mathbf{w}\|_{0}\right)$ plus the power dissipated by digital-to-analog and analog-todigital converters $\left(P_{\mathrm{dac}}, P_{\mathrm{adc}}\right)$ and by $\mathrm{RF}$ chains $\left(P_{\mathrm{rfc}}\right)$ :

$$
\begin{aligned}
P_{\text {data }} & =\|\mathbf{f}\|_{2}^{2}, \\
P_{c} & =\|\mathbf{f}\|_{0} \cdot\left(P_{\mathrm{sw}}+P_{\mathrm{ps}}+P_{\mathrm{pa}}\right)+P_{\mathrm{dac}}+P_{\mathrm{rfc}} \\
& +\|\mathbf{w}\|_{0} \cdot\left(P_{\mathrm{lna}}+P_{\mathrm{sw}}+P_{\mathrm{ps}}\right)+P_{\mathrm{adc}}+P_{\mathrm{rfc}}
\end{aligned}
$$

where $P_{\mathrm{sw}}, P_{\mathrm{ps}}, P_{\mathrm{pa}}, P_{\mathrm{lna}}$ respectively stand for power consumed by switches (during switching transition), phase shifters, PA and LNA [5]. $\|\cdot\|_{p}$ is the $\ell_{p}$-norm. We can rewrite $P_{\text {tot }}$ as

$$
P_{\text {tot }}=\|\mathbf{f}\|_{2}^{2}+b \cdot\|\mathbf{f}\|_{0}+c \cdot\|\mathbf{w}\|_{0}+d \text {, }
$$

where $b \triangleq P_{\mathrm{sw}}+P_{\mathrm{ps}}+P_{\mathrm{pa}}, c \triangleq P_{\mathrm{lna}}+P_{\mathrm{sw}}+P_{\mathrm{ps}}$, and $d \triangleq P_{\mathrm{dac}}+P_{\mathrm{adc}}+2 P_{\mathrm{rfc}}$. Note that $b, c, d>0$.

\section{B. Energy Efficiency at Fixed PER}

The recent literature has made a dedicated effort to optimizing similar EE figures of merit [21]. Therein, the configurations that achieve maximum EE yield moderate transmission rates and low power consumption. Accordingly, we propose the following approach as design guideline: minimize total power consumption subject to an upper bound constraint on the PER.
This is relevant in practice, where the upper bound $0<\epsilon<1$ can be determined for a given application and channel encoder.

Therefore, we impose

$$
\operatorname{PER}[\Gamma] \leq \epsilon,
$$

and proceed to minimize the total end-to-end power consumption $P_{\text {tot }}$ in (19). Note that (20) is equivalent to the following inequality constraint

$$
\frac{\left|\mathbf{w}^{H} \mathbf{H f}\right|^{2}}{\mathbf{w}^{H} \mathbf{w}} \geq \rho,
$$

with $\rho \triangleq \operatorname{PER}^{-1}[\epsilon] \cdot \frac{1}{\gamma}$ a known value given $\epsilon$. An appropriate value for $\epsilon$ that allows neglecting packet retransmissions for system modeling, is to set $\epsilon$ in $\left[10^{-4}, 10^{-3}\right]$.

Before stating the optimization problem of the design outlined above, let us introduce first the following vectors, which combine the effects of the phase shifters and switches:

$$
\begin{aligned}
& \boldsymbol{\varphi}_{t} \triangleq \boldsymbol{\beta}_{t} \odot \boldsymbol{\phi}_{t} \text { with } \varphi_{i}^{t} \triangleq\left[\boldsymbol{\varphi}_{t}\right]_{i} \in\{0\} \cup \mathcal{S}_{t}, \\
& \boldsymbol{\varphi}_{r} \triangleq \boldsymbol{\beta}_{r} \odot \boldsymbol{\phi}_{t} \text { with } \varphi_{i}^{r} \triangleq\left[\boldsymbol{\varphi}_{r}\right]_{i} \in\{0\} \cup \mathcal{S}_{r} .
\end{aligned}
$$

Therefore, we undertake the minimization of $P_{\text {tot }}$ as follows:

$$
\begin{aligned}
\min _{\boldsymbol{\alpha}, \boldsymbol{\varphi}_{t}, \boldsymbol{\varphi}_{r}} & \|\mathbf{f}\|_{2}^{2}+b \cdot\|\mathbf{f}\|_{0}+c \cdot\|\mathbf{w}\|_{0}+d \\
\text { s.t. } & \mathbf{f}=L_{\mathrm{sw}} L_{\mathrm{ps}} \cdot \boldsymbol{\varphi}_{t} \odot \boldsymbol{\alpha} \\
\text { s.t. } & \mathbf{w}=L_{\mathrm{sw}} L_{\mathrm{ps}} \cdot \boldsymbol{\varphi}_{r} \\
\text { s.t. } & \frac{\left|\mathbf{w}^{H} \mathbf{H} \mathbf{f}\right|^{2}}{\mathbf{w}^{H} \mathbf{w}} \geq \rho \\
\text { s.t. } & \alpha_{1 \leq i \leq L_{t}} \in \mathcal{A} \\
\text { s.t. } & \varphi_{1 \leq i \leq L_{t}}^{t} \in\{0\} \cup \mathcal{S}_{t}, \quad \varphi_{1 \leq i \leq L_{r}}^{r} \in\{0\} \cup \mathcal{S}_{r}
\end{aligned}
$$

Since the problem needs the joint design of vectors $\mathbf{f}$ and $\mathbf{w}$, we tackle problem (24a)-(24f) by separating it in two subproblems. We describe both optimization subproblems in the sequel so as to get insight of the optimum beamformers.

1) Combiner optimization for a fixed $\mathbf{f}=\tilde{\mathbf{f}}$ : The optimum $\mathbf{w}$, denoted $\mathbf{w}_{*}$, is the solution to the following subproblem:

$$
\begin{aligned}
\mathbf{w}_{*}=\underset{\mathbf{w}}{\arg \min } & \|\mathbf{w}\|_{0} \\
\text { s.t. } & \mathbf{w}=L_{\mathrm{sw}} L_{\mathrm{ps}} \cdot \boldsymbol{\varphi}_{r} \\
\text { s.t. } & \frac{\left|\mathbf{w}^{H} \mathbf{H} \tilde{\mathbf{f}}\right|^{2}}{\mathbf{w}^{H} \mathbf{w}} \geq \rho \\
\text { s.t. } & \varphi_{1 \leq i \leq L_{r}}^{r} \in\{0\} \cup \mathcal{S}_{r}
\end{aligned}
$$

Thus, the optimal combiner $\mathbf{w}_{*}$ is the sparsest vector with nonzero entries in $\mathcal{S}_{r}$ that achieves the requirement $(25 \mathrm{c})$.

2) Precoder optimization for a fixed combiner $\mathbf{w}=\tilde{\mathbf{w}}$ : We study the design of precoder $\mathbf{f}$ so as to satisfy the SNR requirement and alphabet constraints. Let the combiner be fixed to some value $\mathbf{w}=\tilde{\mathbf{w}}$. The optimal precoder $\mathbf{f}$, denoted $\mathbf{f}_{*}$, is the solution to

$$
\begin{aligned}
& \mathbf{f}_{*}=\underset{\mathbf{f}}{\arg \min }\|\mathbf{f}\|_{2}^{2}+b \cdot\|\mathbf{f}\|_{0} \\
& \text { s.t. } \mathbf{f}=L_{\mathrm{sw}} L_{\mathrm{ps}} \cdot \boldsymbol{\varphi}_{t} \odot \boldsymbol{\alpha} \\
& \text { s.t. }\left|\tilde{\mathbf{w}}^{H} \mathbf{H f}\right|^{2} \geq \rho \cdot \tilde{\mathbf{w}}^{H} \tilde{\mathbf{w}} \\
& \text { s.t. } \boldsymbol{\alpha}_{1 \leq i \leq L_{t}} \in \mathcal{A}, \varphi_{1 \leq i \leq L_{t}}^{t} \in\{0\} \cup \mathcal{S}_{t}
\end{aligned}
$$


It is worth noting that constraints (26a)-(26d) depend on the quantization levels of both phase shifters and PAs. In the sequel, we assume fixed sets $\mathcal{A}$ and $\mathcal{S}_{t}$.

\section{Cyclic Minimization Approach}

In view of Section III-B and the challenging problem of determining the optimal precoder and combiner vectors, we propose an iterative optimization leveraging the cyclic minimization approach sketched in Algorithm 1 (explained below).

For a given channel matrix, we initialize vectors $\alpha, \varphi_{t}$ and $\varphi_{r}$ randomly. In Algorithm 1, superindices denote the iteration index. At each iteration $i$, we find $\alpha, \varphi_{t}$ and $\varphi_{r}$ according to the greedy algorithm VECTORMIN. These steps are repeatedly iterated until convergence. In each VECTORMIN optimization, every element of the input vector $\mathrm{x}$ is individually optimized while fixing the others. Since the set of feasible values for each scalar variable is finite, minimization can be readily done by directly evaluating the cost function at those values.

Note that, depending on the instantaneous channel, it is not always possible to find solutions that attain the PER requirement. In these cases, there is no feasible solution and we decide not to transmit by deactivating all switches.

At each step, the scheme outlined in Algorithm 1 will reduce the cost function, as is true for any cyclic optimization method [25]. Since the cost function is lower bounded, the sequence of cost values produced by Algorithm 1 must be convergent. However, given the non-convex nature of the problem at hand, there is no guarantee that the global optimum will be obtained. A straightforward strategy to improve performance at the price of increased computational complexity is to run the algorithm multiple (say $N_{\text {cyc }}$ ) times with different random initializations, and then pick the best solution. As will be shown in Section IV, in high SNR scenarios it is possible to obtain very competitive results with a reasonable value of $N_{\text {cyc }}$.

\section{Particular Case: High-SNR Analysis}

We analyze a computationally efficient sub-optimal approach valid for sufficiently high values of the pre-combining SNR $\gamma$.

Bearing in mind the typical low-complexity of user terminals, one may seek a simpler optimization problem. Assume that $\gamma$ is large enough so as only one receiving antenna is needed to meet the PER requirement given a fixed precoder $\tilde{\mathbf{f}}$. Thus, the optimization in (25a)-(25d) reduces to an antenna selection problem. In this case, the joint design of analog precoder and combiner is solved as: for $\iota=1, \ldots, L_{r}$, set $\mathbf{w}=\mathbf{e}_{\iota}$ (the $\iota$-th element of the canonical basis) and optimize f so as to guarantee the PER requirement, as follows,

$$
\begin{aligned}
\mathbf{f}_{*}[\iota]=\underset{\mathbf{f}}{\arg \min }\|\mathbf{f}\|_{2}^{2}+b \cdot\|\mathbf{f}\|_{0} \\
\text { s.t. } \mathbf{f}=L_{\mathrm{sw}} L_{\mathrm{ps}} \cdot \boldsymbol{\varphi}_{t} \odot \boldsymbol{\alpha} \\
\text { s.t. }\left|\mathbf{e}_{\iota}^{H} \mathbf{H f}\right|^{2} \geq \rho \\
\text { s.t. } \boldsymbol{\alpha}_{1 \leq i \leq L_{t}} \in \mathcal{A}, \varphi_{1 \leq i \leq L_{t}}^{t} \in\{0\} \cup \mathcal{S}_{t}
\end{aligned}
$$

This problem can be approximately solved in a greedy way by following steps 13 and 14 of Algorithm 1. Among those solutions $\left\{\mathbf{f}_{*}[\iota], \mathbf{e}_{\iota}\right\}$ that meet the constraint $(27 \mathrm{c})$, the one minimizing the power consumption is selected.

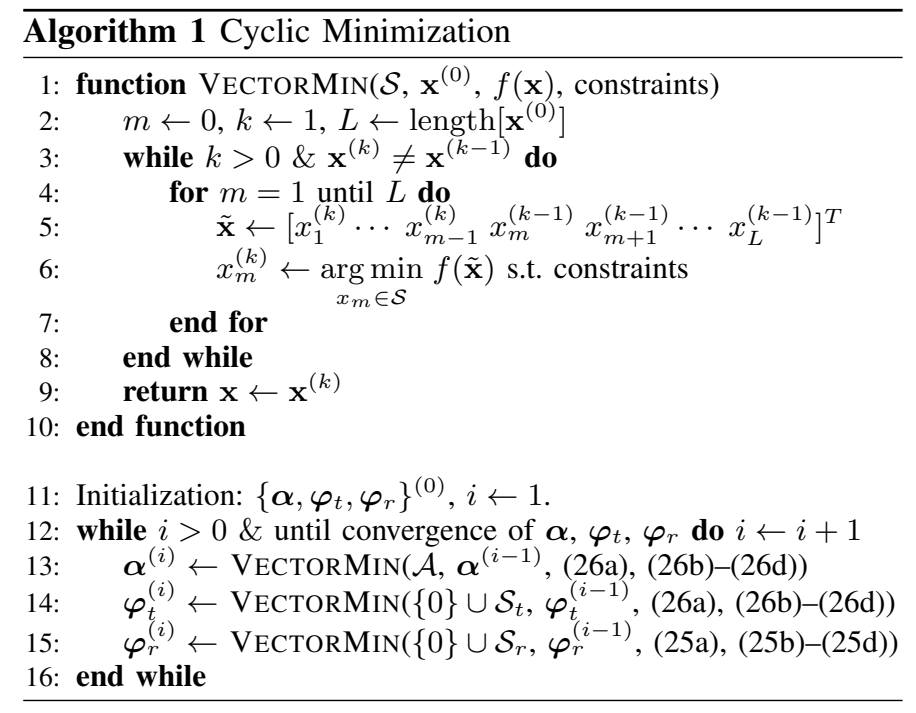

\section{Numerical Results}

We assess the performance of the proposed scheme focusing on devices used in a $60 \mathrm{GHz}$ system with $B=100 \mathrm{MHz}$ bandwidth. For simulations, we consider the transmission of $n$ symbol packets, and we use the large- $n$ second order expansion of the maximal channel coding rate [15] to obtain

$$
\operatorname{PER}[\Gamma]=Q\left(\sqrt{n V^{-1}} \cdot\left(\log _{2}(1+\Gamma)-R\right)\right),
$$

with $V \triangleq\left[1-(1+\Gamma)^{-2}\right] \log _{2}^{2}(e)$ the channel dispersion, and $Q(\cdot)$ the well-known Q-function. We study $n=500$ symbols and $R=1 \mathrm{bit} / \mathrm{s} / \mathrm{Hz}$. The target PER is set to $\epsilon=10^{-3}$, so that, for the evaluated physical layer $\operatorname{PER}^{-1}[\epsilon] \approx 1.02 \mathrm{~dB}$.

With regard to the hardware architecture, we consider the transmitter and the receiver equipped with half-wavelength spaced uniform linear arrays with 4-bit uniformly quantized phase shifters $\left(b_{t}=b_{r}=4\right)$, and PAs with saturation power $P_{0}=30 \mathrm{~mW}$ and able to vary their transmitted power over the $K=10$ levels $\alpha_{1 \leq i \leq K}=\sqrt{P_{0} \cdot i / K}$. Power insertion losses are $L_{\mathrm{ps}}^{2}=-3 \mathrm{~dB}$ [26] and $L_{\mathrm{sw}}^{2}=-1 \mathrm{~dB}$ [5]. Relevant specificities of the power consumed by each element are shown in Table I.

For simulations, we adopt the channel model in [5, eq. (17)] with $N_{\mathrm{cl}}=3$ clusters and $N_{\text {ray }}=7$ rays per cluster. The path gains $\beta_{k, l}$ are independently obtained from a circular complex Gaussian distribution. With regard to the steering vectors $\mathbf{a}_{t}$ and $\mathbf{a}_{r}$, the angles of departure and arrival are uniformly distributed with angular spreads of $\pm \pi / 3$. We remark that all channel matrices have been normalized as $\operatorname{Tr}\left(\mathbf{H}^{H} \mathbf{H}\right)=L_{t} L_{r}$.

In the sequel, we analyze the system performance versus the pre-combining SNR $\gamma$ for several cases. We consider: firstly, full-analog beamforming (Full AB), where transceivers combine phase shifters and switches; and secondly, on-off analog beamforming (On-Off $\mathrm{AB}$ ), where they only use switches. OnOff $A B$ is a particular case of Full $A B$ where we do not have

TABLE I: Power consumption of analog elements. The values are the same as in $\left[5\right.$, p.253], which are normalized by the reference $P_{\text {ref }}=20 \mathrm{~mW}$.

\begin{tabular}{|c|c|c|c|c|c|c|}
\hline$P_{\mathrm{sw}}$ & $P_{\mathrm{ps}}$ & $P_{\mathrm{pa}}$ & $P_{\text {lna }}$ & $P_{\mathrm{adc}}$ & $P_{\mathrm{dac}}$ & $P_{\mathrm{rfc}}$ \\
\hline 0.25 & 1.50 & 1 & 1 & 10 & 10 & 2 \\
\hline
\end{tabular}




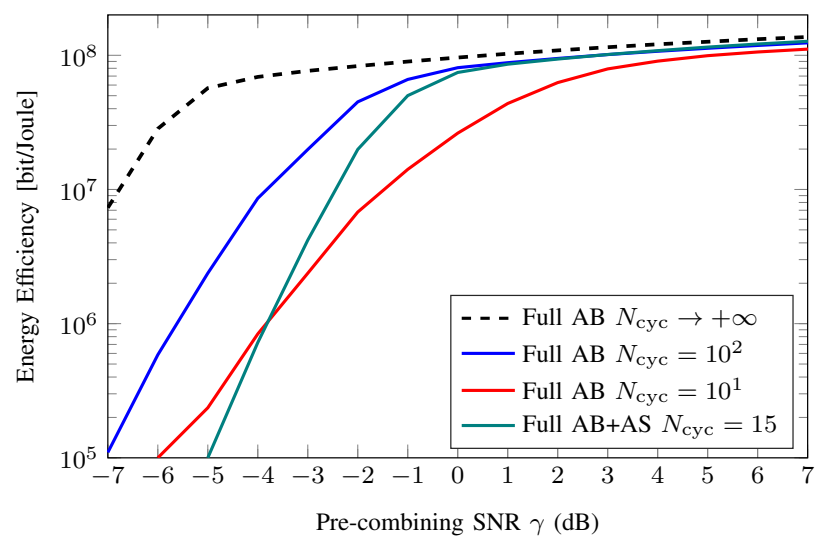

Fig. 2: $\mathrm{EE}$ (15) versus $\gamma$ in the full $\mathrm{AB}$ case. Full $\mathrm{AB}$ with the $\mathrm{AS}$ approach at reception described in Section III-D is evaluated as well.

phase shifters. In both cases, we study Algorithm 1 and the antenna selection (AS) approach presented in Section III-D.

\section{A. System Performance Analysis in terms of EE}

We depict the EE versus $\gamma$ when using Full $\mathrm{AB}$ in Fig. 2 and On-Off $A B$ in Fig. 3. The reason for this analysis is because it highlights the interplay between the cost of power adaptation and that of activating switches at system ends. The EE values obtained with the cyclic minimization approach of Section III-C are shown for $N_{\text {cyc }}=10$ and 100 initializations, as well as for a large value of this parameter. Also shown are the EE values obtained by the AB+AS scheme with $N_{\text {cyc }}=15$ initializations. Results are simulated for $L_{t}=32$ and $L_{r}=8$ antennas. The number of active switches are shown in Fig. 4.

In the low SNR regime (long-range communication), power adaptation compensates for high channel attenuation, so that the system can operate at the desired PER in detriment of EE. The EE severely decays at very low $\gamma$ because, depending on the instantaneous channel conditions, the total available power is not sufficient to compensate for path loss. Thus, it is preferable not to transmit by deactivating all switches. This makes the $\mathrm{EE}$ for that channel realization equal to 0. Full $\mathrm{AB}$ outperforms On-Off $\mathrm{AB}$ because phase shifters, although having larger insertion losses, provide a better coherent combination of signals, thus, increasing the SNR after combining. This is remarkable when the link operates in challenging channel conditions. Note that in the On-Off AB case (Fig. 3), AS reaches a very competitive EE with a reduced complexity.

In the low SNR regime, as seen in Fig. 4, as the SNR increases more switches become activated in order to achieve the PER requirement (20), while reducing the power supplied by each antenna's PA. On the other hand, at moderate/high SNR (short-distance communication), the channel provides more gain to each transmitter-receiver antenna path. Consequently, fewer switches at both communication sides are activated since the PER requirement can be attained with a smaller number of antennas. In this regime, the performance of On-Off $A B$ is similar to that of full $\mathrm{AB}$, so that similar $\mathrm{EE}$ values can be obtained with much less hardware complexity. With respect to AS, higher values of EE are obtained with a reduced $N_{\text {cyc }}$.

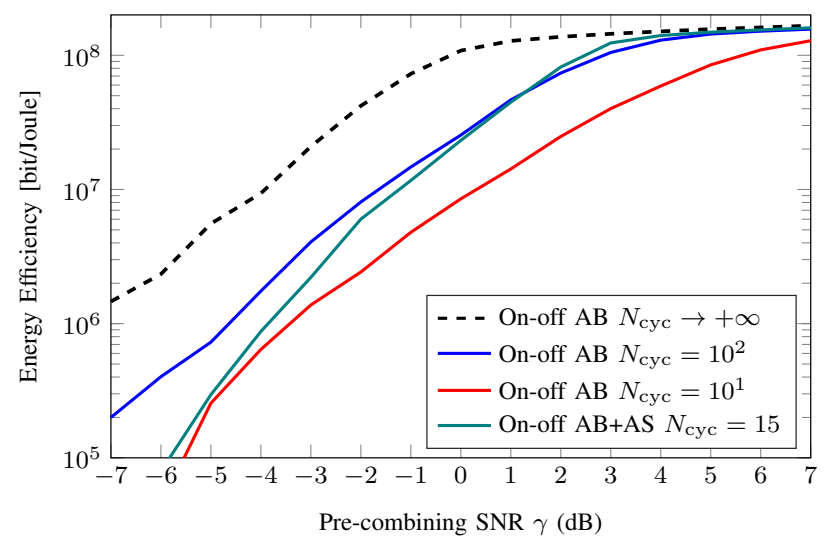

Fig. 3: $\mathrm{EE}$ (15) versus $\gamma$ in the on-off $\mathrm{AB}$ case. On-off $\mathrm{AB}$ with the $\mathrm{AS}$ approach at reception described in Section III-D is evaluated as well.

A last remark on the convergence of cyclic minimization is pointed out next. In the low SNR regime, using large $N_{\text {cyc }}$ significantly improves performance, but as the SNR increases a smaller number of initializations seem to suffice.

\section{B. System Performance Analysis versus Transmit Antennas $L_{t}$}

Figures 5 and 6 illustrate the $\mathrm{EE}$ as a function of the number of transmit antennas $L_{t}$, for $L_{r}=8$ antennas at the receiver. The motivation of such study relies on the ability of large antenna arrays to deliver high diversity gains that can compensate demanding SNR requirements.

As expected, larger values of $L_{t}$ can balance more challenging channel conditions. Notwithstanding, as we analyze the EE subject to a given channel encoder, the EE practically saturates for some $L_{t}$. The latter is better highlighted in the full $\mathrm{AB}$ case, where phase shifters transfer power in a better way by (almost) compensating the channel phases. Instead, in the On-Off AB case, EE saturation is experimented at higher $L_{t}$ values with respect to full $\mathrm{AB}$ at equal $N_{\text {cyc }}$.

In short, the larger the number of antennas, the larger the number of possible channels. Thus, under the assumption of full CSI, the optimum configuration selects the minimum number of channels necessary to meet the PER requirement.

\section{CONCLUSION}

We have addressed the design of mmWave analog precoder and combiner from an energy efficiency perspective. We have considered a point-to-point scenario under a single channel encoder and terminals handling analog beamformers that combine phase shifters, switches and variable-gain power amplifiers. The challenging problem has been solved firstly, by mapping the physical layer characteristic to a fixed signal-to-noise ratio point that allows an acceptable system error rate, and secondly, minimizing the total end-to-end consumed power. A cyclic algorithm has been used to tackle system optimization under discrete alphabets due to hardware impairments. Simulations have shown that the full beamforming case outperforms in noise-limited scenarios whereas the on-off beamforming is optimal in high SNR situations. In the latter case, we assess that antenna selection at the receiver allows a competitive configuration in terms of $\mathrm{EE}$ and complexity. 


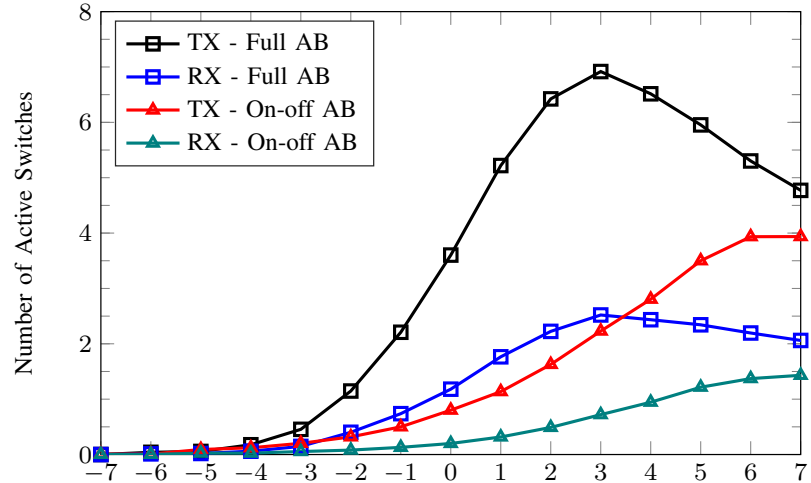

Pre-combining SNR $\gamma(\mathrm{dB})$

Fig. 4: Number of active switches, at the transmitter and the receiver, versus $\gamma$ in full $\mathrm{AB}$ and on-off $\mathrm{AB}$ cases. Results are computed under $N_{\text {cyc }}=10^{2}$.

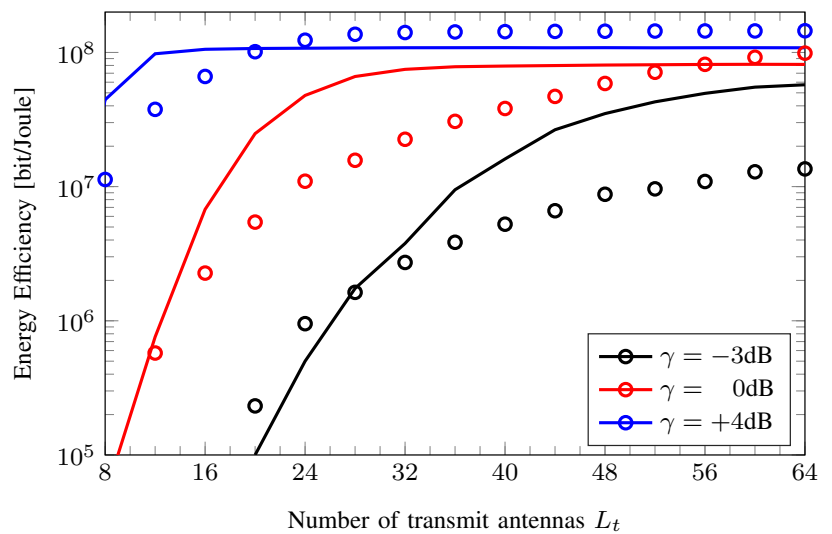

Fig. 6: EE (15) versus $L_{t}$. Full AB+AS are depicted in solid lines whereas onoff $\mathrm{AB}+\mathrm{AS}$ are shown using markers. Results are computed under $N_{\text {cyc }}=15$.

\section{REFERENCES}

[1] Y. He, J. Guo, and X. Zheng, "From surveillance to digital twin: Challenges and recent advances of signal processing for industrial internet of things," IEEE Signal Process. Mag., vol. 35, no. 5, pp. 120 129, Sep. 2018.

[2] Z. Dawy, W. Saad, A. Ghosh, J. G. Andrews, and E. Yaacoub, "Toward massive machine type cellular communications," IEEE Wireless Commun., vol. 24, no. 1, pp. 120-128, Feb. 2017.

[3] J. G. Andrews, S. Buzzi, W. Choi, S. V. Hanly, A. Lozano, A. C. K. Soong, and J. C. Zhang, "What will 5G be?," IEEE J. Sel. Areas Commun., vol. 32, no. 6, pp. 1065-1082, Jun. 2014.

[4] R. W. Heath, N. González-Prelcic, S. Rangan, W. Roh, and A. M. Sayeed, "An overview of signal processing techniques for millimeter wave MIMO systems," IEEE J. Sel. Topics Signal Process., vol. 10, no. 3, pp. 436453, Apr. 2016.

[5] R. Méndez-Rial, C. Rusu, N. González-Prelcic, A. Alkhateeb, and R. W. Heath, "Hybrid MIMO architectures for millimeter wave communications: Phase shifters or switches?," IEEE Access, vol. 4, pp. 247-267, Jan. 2016.

[6] A. Alkhateeb, G. Leus, and R. W. Heath, "Limited feedback hybrid precoding for multi-user millimeter wave systems," IEEE Trans. Wireless Commun., vol. 14, no. 11, pp. 6481-6494, Nov. 2015.

[7] D. H. N. Nguyen, L. B. Le, T. Le-Ngoc, and R. W. Heath, "Hybrid MMSE precoding and combining designs for mmWave multiuser systems," IEEE Access, vol. 5, pp. 19167-19181, Sep. 2017.

[8] J. Jin, Y. R. Zheng, W. Chen, and C. Xiao, "Hybrid precoding for millimeter wave MIMO systems: A matrix factorization approach," IEEE Trans. Wireless Commun., vol. 17, no. 5, pp. 3327-3339, May 2018.

[9] Z. Wang, M. Li, Q. Liu, and A. L. Swindlehurst, "Hybrid precoder and combiner design with low-resolution phase shifters in mmWave MIMO systems," IEEE J. Sel. Topics Signal Process., vol. 12, no. 2, pp. 256269, May 2018.

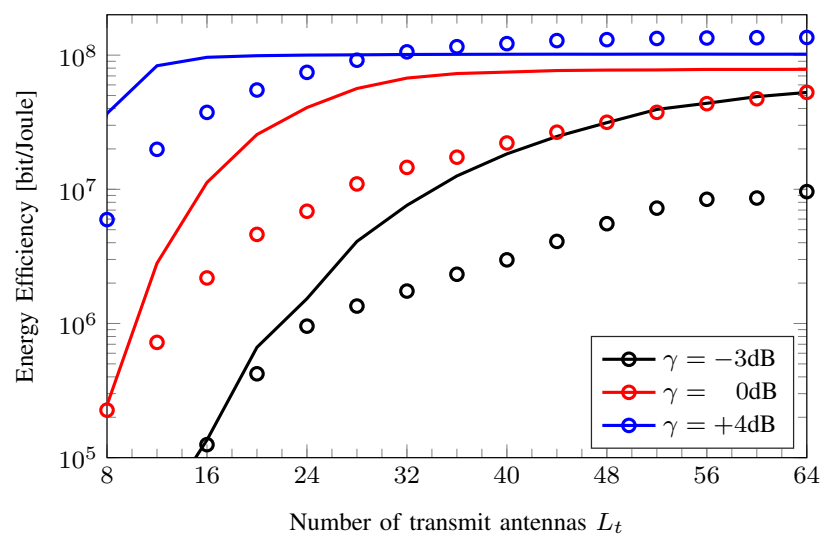

Fig. 5: EE (15) versus $L_{t}$. Full AB are depicted in solid lines whereas on-off $\mathrm{AB}$ are shown using markers. Results are computed under $N_{\mathrm{cyc}}=10^{2}$.

[10] J. Rodríguez-Fernandez, R. López-Valcarce, and N. González-Prelcic, "Frequency-selective hybrid precoding and combining for mmwave MIMO systems with per-antenna power constraints," in 2019 IEEE Int. Conf. Acoust., Speech and Signal Process., May 2019, pp. 4794-4798.

[11] S. Park, A. Alkhateeb, and R. W. Heath, "Dynamic subarrays for hybrid precoding in wideband mmWave MIMO systems," IEEE Trans. Wireless Commun., vol. 16, no. 5, pp. 2907-2920, May 2017.

[12] J. P. González-Coma, R. Maneiro-Catoira, and L. Castedo, "Hybrid precoding with time-modulated arrays for mmwave MIMO systems," IEEE Access, vol. 6, pp. 59422-59437, 2018.

[13] S. Zhang, C. Guo, T. Wang, and W. Zhang, "ON-OFF analog beamforming for massive MIMO," IEEE Trans. Veh. Technol., vol. 67, no. 5, pp. 4113-4123, May 2018.

[14] F. Molina and J. Borras, "Low-complexity switching network design for hybrid precoding in mmWave MIMO systems," in 2019 27th European Signal Process. Conf. (EUSIPCO), Sep. 2019.

[15] Y. Polyanskiy, H. V. Poor, and S. Verdú, "Channel coding rate in the finite blocklength regime," IEEE Trans. Inf. Theory, vol. 56, no. 5, pp. 2307-2359, May 2010.

[16] S. Payami, M. Ghoraishi, and M. Dianati, "Hybrid beamforming for large antenna arrays with phase shifter selection," IEEE Trans. Wireless Commun., vol. 15, no. 11, pp. 7258-7271, Nov. 2016.

[17] D. Zhang, Y. Wang, X. Li, and W. Xiang, "Hybridly connected structure for hybrid beamforming in mmwave massive MIMO systems," IEEE Tran. Commun., vol. 66, no. 2, pp. 662-674, Feb. 2018.

[18] S. Payami, M. Ghoraishi, M. Dianati, and M. Sellathurai, "Hybrid beamforming with a reduced number of phase shifters for massive MIMO systems," IEEE Trans. Veh. Tech., vol. 67, no. 6, pp. 4843-4851, Jun. 2018.

[19] X. Gao, L. Dai, S. Han, C. I, and R. W. Heath, "Energy-efficient hybrid analog and digital precoding for mmwave MIMO systems with large antenna arrays," IEEE J. Sel. Areas Commun., vol. 34, no. 4, pp. 9981009, Apr. 2016.

[20] S. He, C. Qi, Y. Wu, and Y. Huang, "Energy-efficient transceiver design for hybrid sub-array architecture MIMO systems," IEEE Access, vol. 4, pp. 9895-9905, 2016.

[21] S. Payami, N. Mysore Balasubramanya, C. Masouros, and M. Sellathurai, "Phase shifters versus switches: An energy efficiency perspective on hybrid beamforming," IEEE Wireless Commun. Lett., vol. 8, no. 1, pp. 13-16, Feb. 2019.

[22] K. F. Trillingsgaard and P. Popovski, "Encoding of control information and data for downlink broadcast of short packets," in 2016 Inf. Theory and Applications Workshop (ITA), Jan. 2016, pp. 1-5.

[23] G. Durisi, T. Koch, and P. Popovski, "Toward massive, ultrareliable, and low-latency wireless communication with short packets," Proc. IEEE, vol. 104, no. 9, pp. 1711-1726, Sep. 2016.

[24] X. Chen, D. Wing Kwan Ng, W. Yu, E. G. Larsson, N. Al-Dhahir, and R. Schober, "Massive access for 5g and beyond," 2020.

[25] P. Stoica and Y. Selén, "Cyclic minimizers, majorization techniques, and the expectation-maximization algorithm: A refresher," IEEE Signal Process. Mag., vol. 21, no. 1, pp. 112-114, Feb. 2004.

[26] G. Shin, J. Kim, H. Oh, S. Choi, C. W. Byeon, J. H. Son, J. H. Lee, and C. Kim, "Low insertion loss, compact 4-bit phase shifter in $65 \mathrm{~nm}$ CMOS for 5G applications," IEEE Microw. Wireless Compon. Lett., vol. 26, no. 1, pp. 37-39, Jan. 2016. 